\section{Facharztprüfung zur Erlangung des Facharzttitels FMH für Pathologie}

Aufgrund des Weiterbildungsprogrammes und des Beschlusses des Zentralvorstandes der FMH vom 3. Februar 1999 ist das Bestehen der Facharztprüfung für alle Kandidatinnen und Kandidaten, welche ihre Weiterbildung nach dem 31. Dezember 2000 abschliessen, Voraussetzung zur Erlangung des Facharzttitels FMH für Pathologie. Näheres zu den Übergangsbestimmungen und Ausnahmen ist in der Schweizerischen Ärztezeitung Nr. 9 vom 3. März 1999 publiziert.

\section{Frühjahrssession 2001}

Ort: Institut Universitaire de Pathologie, rue du Bugnon 25, 1011 Lausanne

Datum: Freitag und Samstag, 27. und 28. April 2001

Anmeldefrist: 10. März 2001
Herbstsession 2001

Ort: Pathologisches Institut der Universität Bern, Murtenstrasse 31, 3010 Bern

Datum: Freitag und Samstag, 26. und 27. Oktober 2001

Anmeldefrist: 15. September 2001

$\Rightarrow$ Wichtig: Die Teilnehmerzahl ist auf 6 pro Session begrenzt. Die Anmeldungen werden in der Reihenfolge ihres Eintreffens berücksichtigt!

Prüfungsgebühr: Fr. 300.- für Mitglieder der Schweizerischen Gesellschaft für Pathologie, Fr. 450.- für Nichtmitglieder

Anmeldeformulare können bezogen werden bei: Prof. C. Y. Genton

Institut Universitaire de Pathologie

25, rue du Bugnon, 1011 Lausanne

Tel. 02131471 20, Fax 0213147115

E-mail claude.genton@chuv.hospvd.ch

\section{Examen de spécialiste pour l'obtention du titre FMH en Pathologie}

Conformément au programme de formation postgraduée et à la décision du Comité central de la FMH du 3 février 1999, la réussite de l'examen de spécialiste est une condition requise pour les candidats au titre FMH en Pathologie qui termineront leur formation postgraduée après le 31 décembre 2000 . Pour de plus amples renseignements concernant les dispositions transitoires et les dérogations, veuillez vous référer à la publication du Bulletin des médecins suisses no 9, du 3 mars 1999.

\section{Session de printemps 2001}

Lieu: Institut Universitaire de Pathologie, rue du Bugnon 25, 1011 Lausanne

Date: Vendredi et samedi, 27 et 28 avril 2001

Délai d'inscription: 10 mars 2001
Session d'automne 2001

Lieu: Pathologisches Institut der Universität Bern, Murtenstrasse 31, 3010 Bern

Date: Vendredi et samedi, 26 et 27 octobre 2001

Délai d'inscription: 15 septembre 2001

$\Rightarrow$ Important: Le nombre de participants est limité à 6 par session. Les inscriptions seront validées dans l'ordre de leur réception!

Taxe d'examen: Fr. 300.- pour les membres de la Société Suisse de Pathologie, Fr. 450.- pour les nonmembres

Les formulaires d'inscription peuvent être obtenus auprès du:

Prof. C. Y. Genton

Institut Universitaire de Pathologie 25, rue du Bugnon, 1011 Lausanne tél. 02131471 20, fax 0213147115 e-mail claude.genton@chuv.hospvd.ch 